\title{
Mujer y cimarronaje en la región histórica de Vueltabajo, Cuba (1790-1850)
}

Jorge Freddy Ramirez Pérez

Universidad de Pinar del Rio (Cuba)

Pedro Luis Hernández Pérez

Unión Nacional de Escritores y Artistas de Cuba, UNEAC (Cuba)

Silfredo Rodriguez Basso

Universidad de Pinar del Rio (Cuba) 



\title{
Mujer y cimarronaje en la región histórica de Vueltabajo, Cuba (1790-1850)
}

\section{Woman and cimarronaje in the historical region of Vueltabajo, Cuba (1790-1850)}

\author{
Jorge Freddy Ramirez Pérez \\ Universidad de Pinar del Rio (Cuba) \\ freddy@upr.edu.cu
}

\section{Pedro Luis Hernández Pérez}

Unión Nacional de Escritores y Artistas de Cuba, UNEAC (Cuba)

pedro@gmail.com

\author{
Silfredo Rodriguez Basso \\ Universidad de Pinar del Rio (Cuba) \\ antropol@upr.edu.cu
}

Fecha de recepción: 10 de octubre de 2018

Fecha de aceptación: 5 de abril de 2019

\section{Resumen}

El presente artículo, aborda una mirada de género al interior de la resistencia esclava en la región histórica de Vueltabajo, en Cuba. Se establecen los parámetros geohistóricos, económicos y naturales que condicionan la presencia de la mujer cimarrona, con dos estudios de casos representativos: el de la Madre Melchora y el de Petrona Conga. Los métodos histórico-lógico, y de recopilación, ordenamiento y análisis documental de las fuentes primarias, sustentaron los resultados que se presentan. Se resalta a modo de conclusiones, el papel de las mujeres en el cimarronaje como portadoras y trasmisoras de una cultura de resistencia, lo que contrasta con la escasa atención que sobre el asunto existe en la historiografía cubana.

Palabras clave: Género; Esclavitud; Resistencia; Palenque; Rancheador; Cimarronaje; Cuba

\footnotetext{
Abstract

This article reports a gender perspective within the slave resistance in the historical region of Vueltabajo, in Cuba. The geohistorical, economic and natural parameters that determine the presence of the Maroon woman are established, with two representative case studies: that of Mother Melchora and that of Petrona Conga.
} 
Mujer y cimarronaje en la región histórica... - J. F. Ramirez Pérez y otros

The historical-logical methods, and the collection, ordering and documentary analysis of the primary sources, supported the results presented. It highlights as a conclusion, the role of women in the cimarronaje as carriers and transmitters of a culture of resistance, which contrasts with the scant attention on the matter in Cuban historiography.

Keywords: Gender; Slavery; Resistance; Palenque; Rancheador; Cimarronaje; Cuba

\section{INTRODUCGIÓN}

La presencia de la mujer en los acontecimientos de la historia cubana, ha cobrado vigor en los estudios historiográficos de los últimos tiempos. Quizás el hecho menos tratado está relacionado con la participación de las mujeres esclavas africanas en las diferentes manifestaciones de resistencia contra el sistema esclavista.

Este fenómeno no se produjo solo en Cuba, sino también en todas las colonias donde se introdujo la mano de obra esclava. La participación femenina, en las diferentes formas de resistencia, posee sus particularidades, además de estar en correspondencia con los escenarios donde se produjeron, en los que tuvo mucho que ver su condición de género, cuyas diferencias -en las que predominó el hombredeterminaron la forma en que ella ejerció este rol.

En el caso de la mujer cimarrona, en general, su condición estaba estrechamente relacionada con el interés de los hombres cimarrones en tener mujeres en palenques y rancherías como esposas, amas de casa y procreadoras de sus hijos, sin descartar la de objeto sexual (Thompson, 2005).

No pocas mujeres huyeron con sus hijos e incluso, las embarazadas, con el fin de evitar que sus hijos nacieran en cautiverio. Son varios los casos de hijos nacidos en condiciones de cimarronaje, tanto en Cuba como en el resto de las colonias en América donde se ha realizado estudios al respecto, como en Colombia, Venezuela, Haití, Surinam, entre otros (Thompson, 2005).

Aunque no existen estadísticas que muestren el peso de la mujer en las comunidades cimarronas, para el caso de Cuba, y en particular la región de Vueltabajo, se hace mención con frecuencia, en las fuentes existentes, de la presencia femenina en mayor o menor proporción; no obstante, existen estadísticas para los palenques de la región de Oriente, como por ejemplo, el "Todos tenemos," donde el número de mujeres era similar al de los hombres, y el del "Frijol", donde las féminas superaban a los varones (Thompson, 2005).

En el resto de las colonias existen evidencias del mismo fenómeno en el siglo XVII, pero en casos puntuales como en Colombia, Haití y República Dominicana, donde se reportan un número considerable de mujeres, cerca de un $45 \%$ con respecto a los hombres. Para fines del siglo XVIII, en la colonia francesa de Guadalupe, 
Revista de Humanidades, 37 (2019).p. 73-98. ISSN 1130-5029

la proporción era de una mujer por cada dos hombres cimarrones; y en Haití, el estimado era de un 15 a $20 \%$ de mujeres. En el caso de los estados del sur de Estados Unidos de Norteamérica, se calcula entre un 10 y $12 \%$. Para Surinam y Colombia, un $10 \%$ (Thompson, 2005).

En el caso de Cuba, y, específico en la región histórica de Vueltabajo, el desarrollo económico de finales del siglo XVIII, sobre todo durante la primera mitad de la siguiente centuria, basado en la plantación comercial, trajo consigo la introducción de miles de esclavos africanos, cuya consecuencia inmediata produjo expresiones de resistencia esclava. La misma, se manifestó de diferentes maneras; una de las más radicales fue la conocida como cimarronaje.

Su práctica en Vueltabajo fue significativa, convirtiéndole en una de las regiones de mayor cifra de cimarrones en Cuba. En esta manifestación, no solo los hombres se sumaron a ella, también muchas mujeres enclavas decidieron evadirse de las plantaciones.

Al estudiar la naturaleza de la región vueltabajera, su ubicación geográfica y los hallazgos arqueológicos, se puede tener presente la magnitud de los diversos obstáculos enfrentados por las mujeres cimarronas. El áspero y húmedo bosque, las recónditas montañas de la cordillera de Guaniguanico, las apartadas cuevas, los manglares infestados de plagas, las carencias de alimentos y agua, son algunos de los escollos.

Para la mujer cimarrona, en condiciones de acoso, no solo la naturaleza y el asedio de los rancheadores estaba contra ella, sino también las costumbres ancestrales que las subordinaban al hombre. En algunos de los testimonios documentales de las cimarronas capturadas, aparece la mención de haber sido raptadas o llevadas en contra de su voluntad a los palenques y rancherías, donde eran convertidas en pareja de alguno de los miembros de estas formas asociativas.

En circunstancias de cimarronaje hubo mujeres embarazadas, o huyeron de sus haciendas en plena gestación, sorprendiéndole el parto en los sitios escogidos para refugiarse. La crianza de los infantes en esas circunstancias debió ser muy difícil. Algunos de los cimarrones capturados a lo largo del periodo de su existencia, nacieron y crecieron en ese estado de autoemancipación.

Las mujeres jugaron una función importante en los palenques y rancherías, al ser empleadas, entre otras ocupaciones, como vigías con el fin de proteger los asentamientos. Muchos miembros de los palenques o rancherías, se salvaban gracias al oportuno aviso de estas mujeres.

El presente trabajo ha sido estructurado en varios aspectos, para comprender el contexto espacial y temporal donde se produjo el fenómeno del cimarronaje, y, en particular, la presencia de las mujeres rebeldes. Es la región histórica de Vueltabajo, el escenario geográfico donde se evidencia esta expresión de lucha antiesclavista. 
Uno de sus aspectos, está relacionado con la situación de la región histórica de Vueltabajo, tanto en sus componentes naturales, como en las actividades humanas relacionadas con la cimarronería. En un primer momento, se caracterizan los principales escenarios donde se produjo la mayor presencia de esclavos africanos, así como las zonas escogidas por este componente étnico para recuperar su anhelada libertad.

Se tratan, además, los elementos conformadores del marco histórico en el que se desarrolló el cimarronaje. En él se ponen de relieve, los factores concurrentes y condicionantes de la aparición y desarrollo de la cimarronería, en respuesta a la intensidad que tuvo la esclavitud africana en Cuba.

Un apartado necesario, lo ocupan los componentes identificadores de la cimarronería como práctica de resistencia esclava en la región vueltabajera. No se puede dejar de mencionar cómo esta se produjo, para poder entender el peso específico de la presencia de la mujer cimarrona dentro de este fenómeno.

La última parte del trabajo se vincula de modo directo con la mujer cimarrona, primero evocando algunos testimonios generales que demuestran su presencia a lo largo de medio siglo de cimarronaje en Vueltabajo. De igual manera, se tuvo cuidado en presentar los casos de dos mujeres cimarronas, el de Petrona Conga y la Madre Melchora, cada una de ellas con una historia que evidencia en grado sumo la perspectiva de género de la cimarronería.

Es propósito final del texto, reivindicar a la mujer cimarrona, por su escasa presencia en la historiografía en general, cuya propensión a la masculinidad minimiza en grado considerable la presencia de este sector en las luchas libertarias, sobre todo, en el Caribe. Los nombres de Petrona Conga, la madre Melchora y la madre Pastora, y de otras tantas, quienes dejaron sus restos diseminados por los sitios más recónditos de la geografía cubana, por lo que deben ocupar un lugar digno en la memoria popular de los pueblos.

\section{REGION DE VUELTABAJO EN EL CONTEXTO DE LA ESGLAVI-} TUD

Para poder comprender el proceso evolutivo de la resistencia esclava en la región histórica de Vueltabajo, en particular de su forma más característica, el cimarronaje, es imprescindible el análisis integrador, donde el decurso histórico ha de verse en su estrecha relación espacial. ${ }^{1}$

1 Jorge Freddy Ramírez Pérez y Pedro Luís Hernández Pérez. Regionalización paisajística de la resistencia esclava en Vuelta Abajo, [inédito]. Trabajo presentado en el Congreso Nacional de Historia, Matanzas, 2005 
El papel del espacio geográfico, en su relación con la sociedad, es significativo. En virtud de ello, a cada medio natural, le ha correspondido determinada forma de vínculo desde todas sus dimensiones concurrentes. Las diferentes manifestaciones de resistencia de los esclavos africanos contra el régimen de servidumbre en Cuba, durante la etapa colonial, no quedan exentas de dicho fenómeno.

El escenario donde se produjeron las manifestaciones de cimarronaje, considerado una región histórica, se conoce con el nombre de Vueltabajo, extenso territorio localizado al occidente de la ciudad de La Habana. Sus límites no responden a una estructura político-administrativa, sino a puntos de referencia (sobre todo durante la etapa colonial), dado por geógrafos, viajeros, agrimensores y vecinos en general, a partir de la conformación de un espacio continuo con rasgos comunes.

En la conformación de esta región han incidido diversos factores de diferente procedencia. Entre los accidentes naturales y referentes culturales, adoptados con mayor frecuencia como límites orientales se hallaban: la laguna de Arigüanabo y el límite este de la jurisdicción de Guanajay. También los factores económicos y socioculturales, junto a los naturales, le dieron una configuración particular dentro del contexto nacional.

A partir de estos elementos, el estudio se centra en el espacio comprendido entre los referidos lugares, hasta el cabo de San Antonio, el punto más occidental del archipiélago cubano. Ello se hace desde el criterio de que el fenómeno vinculado a la cimarronería, se manifestó dentro de parámetros geohistóricos y no políticoadministrativos; lo cual ayuda a su comprensión.

La vasta extensión, situada entre los puntos de referencia, está compuesta por varias subregiones naturales, a saber: cordillera de Guaniguanico y las llanuras: meridional, septentrional, y cársica de Guanahacabibes. En ellas, han tenido lugar diferentes tipos de manifestaciones de resistencia activa esclava, muy vinculadas a sus características.

\subsection{Ocupación del espacio}

En este escenario natural, la ocupación humana del espacio en los últimos cuatrocientos años dejó una huella histórica. La conquista y colonización de Cuba impuso una nueva forma de explotación económica a Vueltabajo. Este espacio geográfico tuvo una influencia directa de la expansión económica de la ciudad de $\mathrm{La}$ Habana como punto nodal, lugar donde radicaban los grupos de poder de la sociedad cubana.

Entre los siglos XVI y XVIII, la región mantuvo su unidad geoeconómica, con predominio del sistema de haciendas ganaderas, extendidas por casi todo el territorio. En el caso del tabaco, se produjo una temprana penetración de este cultivo, 
sobre todo por toda la llanura sur. Estas actividades económicas, no produjeron un poblamiento numeroso y mucho menos, nucleado.

Los cambios en el modo de producción, configurados a finales del siglo XVIII, e inicios de la siguiente centuria, originaron la crisis de la primitiva economía ganadera para dar paso a formas superiores y a estructuras sociales más complejas. La de tipo mercantil, basada en la explotación de las plantaciones de azúcar y café, trajo consigo la gradual sustitución de esa estructura secular, sobre todo en el extremo oriental de la región.

A finales del siglo XVIII, la llanura del norte comenzó a ser invadida por la industria azucarera, la que se extiende de forma gradual por el oeste, hasta más allá del pueblo de Bahía Honda. Este proceso coincidió con hechos internacionales de orden político, entre ellos la revolución en la colonia francesa de Saint Domingue (Haití), impulsora de una inmigración forzosa hacia Cuba de personas calificadas, lo que estimuló la economía insular.

Una de las consecuencias inmediatas fue el asentamiento de núcleos étnicos de origen francés en la sierra del Rosario, a inicios del siglo XIX. Este componente, llegó a fundar más de cien plantaciones cafetaleras en sus elevaciones. Estableciendo una verdadera civilización cafetalera.

De igual modo, el extremo oriental de las llanuras septentrional y meridional, fueron invadidas por ingenios y cafetales. Era el momento de los cambios acelerados en la Isla, donde no solo influyeron factores de tipo político sino también de otra índole: la acumulación del capital, los cambios en la estrategia de la metrópoli, sobre todo después de la toma de La Habana por los ingleses, el enraizamiento del iluminismo y del despotismo ilustrado, la revolución industrial y filosófica, y el fortalecimiento de la clase criolla pudiente.

En las tres primeras décadas del siglo XIX, la plantación gozó de gran desarrollo; café y caña de azúcar se adueñaron del paisaje rural del oriente vueltabajero. Como efecto inmediato, ocurre una explosión demográfica en esa porción de la indicada región.

Al contrario, el extremo occidental, continuó su actividad ganadera, pero con un incremento paulatino de la actividad tabacalera; todo lo cual, debido a sus características de manejo, no produjo cambios de consideración desde el punto de vista demográfico en la población esclava, si se compara con la porción levantina.

Como consecuencia, el número de esclavos aumentó en proporción geométrica en la región plantacionista, así como se agudizaron las condiciones precarias de sus vidas. En cambio, en la vega de tabaco, o en sitios de crianza, la mano de obra esclava estuvo limitada a un escaso número por unidad; sus condiciones de vida (correspondiente a una esclavitud más o menos patriarcal) fueron superiores a las del esclavo de la plantación, sin que ello los liberase de la sumisión y los castigos. 
Por lo antes señalado, y a partir de las características geográficas del espacio donde tuvieron lugar las transformaciones económico-sociales referidas, se puede indicar la conformación, hacia la primera mitad del siglo XIX, de varias subregiones geoeconómicas o históricas dentro de la región histórica de Vueltabajo.

\section{CIMARRONAJE EN VUELTABAJO}

En la historia de la región de Vueltabajo, aflora dentro de sus acontecimientos más memorables, la resistencia esclava en sus diversas manifestaciones. Dentro de estas, el cimarronaje se muestra ante el sistema esclavista, como la respuesta de mayor significación.

En este panorama emergen como principales actores los esclavos evadidos, los hacendados y su principal brazo represivo, los rancheadores. Estos hombres, usados contra la indocilidad esclava, no pueden ser suprimidos de los estudios históricos, pues se cercenaría un componente consustancial de ese momento. Cimarrón, hacendado y rancheador, forman parte del triángulo adverso llamado esclavitud.

\subsection{Cimarronaje y medio natural}

El cimarronaje, se produjo a lo largo y ancho del espacio de la región histórica de Vueltabajo, en cada uno de los paisajes que la caracterizan. En cada uno de ellos se efectuó, a modo de tendencia, un tipo de actividad subsistencial, condicionada en unos casos por las características físico-geográficas del territorio donde operaban, y en otros por el grado de control esclavista.

En Vueltabajo, como sucedió en el resto del país, se produjeron tres tipos de manifestaciones de cimarronaje en cuanto a formas de organizarse y operar: cimarronaje simple, en cuadrilla y el palenque. Autores como Franco (1967), con su artículo Cuatro Siglos de Lucha por la Libertad: Los Palenques, La Rosa y González (2004), con su libro Cazadores de Esclavos. Diarios, al igual que Abreu (2012), con Indios Feroces de la Vueltabajo, adoptan esta clasificación.

El cimarronaje simple estuvo relacionado con la huida de un solo hombre por espacio de cierto tiempo. Dicha condición lo obligaba a merodear cerca de las haciendas para poder lograr la subsistencia a partir del robo, u otras formas de obtención de medios necesarios.

Por su parte, el cimarronaje en cuadrilla constituyó una forma de agrupamiento que superó el cimarronaje simple en Vueltabajo. Se caracterizó por la unión de varios cimarrones sin importan su origen etnocultural bajo la dirección de un líder hábil.

En cuanto al apalencamiento, y a diferencia del cimarrón simple y de la cuadrilla de cimarrones, fue una manera de organización más compleja, y a la vez 
superior a las anteriores formas de agrupamiento analizadas. Se identificó por una mayor estabilidad en los lugares escogidos, la reproducción de ciertas maneras de vida africanas adaptadas al contexto vueltabajero, una organización social donde se establecían familias y la consecuente y natural reproducción

Estudios documentales y arqueológicos en Pinar del Río, posibilitan reportar un sin número de sitios vinculados a la cimarronería, los mismos permiten conocer las evidencias materiales de la vida y actividades de los cimarrones, así como los tipos de paisajes donde se desarrollaron estas prácticas de resistencia esclava. De los principales escenarios naturales se pueden conocer sus características.

La sierra del Rosario, se encuentra situada en el extremo oriental de la Cordillera de Guaniguanico, separada de la Sierra de los Órganos por el río San Diego. Constituye una estructura geológica en forma de cadenas montañosas casi paralelas. Las laderas presentan muchas veces pendientes bruscas con cimas puntiagudas, con cuencas y sistemas subterráneos.

Las vías de tránsito natural de la sierra del Rosario, son los profundos valles fluviales que drenan tanto al sur como al norte. En dicha serranía, la tendencia fue hacia el cimarronaje en cuadrillas, cuyos individuos procedían en su mayoría de las dotaciones de ingenios y cafetales.

Este tipo de agrupamiento le permitió moverse de modo constante en una región controlada por los hacendados, con la presencia de rancheadores y otros grupos represivos, organizados para tratar de neutralizarlos. Su estructura organizativa era muy elemental, también existieron en ella manifestaciones de cimarronaje simple y apalencamiento; pero nunca superaron a la cuadrilla.

El cimarronaje en cuadrilla fue en la sierra del Rosario, la táctica más eficaz, pues al no poder estabilizar un tipo de economía propia, basada en la explotación de la tierra, la subsistencia dependía del saqueo de las plantaciones más cercanas a su zona de influencia. Se estableció así una dependencia económica de la cuadrilla alrededor de las haciendas cafetaleras, ingenios y sitios de crianza próximos.

Por su parte, la sierra de los Órganos, la parte más occidental de la cordillera de Guaniguanico, constituye un fenómeno particular de la topografía de Vueltabajo. Sus elevaciones conforman un relieve singular en forma de torres. Su aparato cársico presenta un gran desarrollo, el cual ha contribuido a la formación de un sistema de cavernas y ríos subterráneos, que atraviesan macizos enteros hasta desembocar en las llanuras.

La topografía antes explicada, la existencia de numerosas cuevas y abrigos rocosos con condiciones para habitación humana, así como el aislamiento, al asentarse en hoyos de sierra con tierras fértiles, permitían establecer una economía de subsistencia, $\mathrm{y}$, en consecuencia, la organización de palenques, adaptados a las características propias del medio. Además de ser una zona menos frecuentada por 
los rancheadores, debido, entre otras, cosas a una menor población del territorio, así como mayor inaccesibilidad del macizo montañoso.

Así los apalencados respetaron los principios más elementales de una subsistencia acosada, en primer lugar, la distancia entre el lugar escogido y los núcleos de población y vías de comunicación, primeras regularidades de este tipo de asentamiento humano.

En segundo lugar, los sitios de habitación, como ya se dijo, eran lugares poco accesibles al transeúnte, difíciles de alcanzar, escenarios a los que solo se podía llegar con objetivos muy definidos; y, en tercer lugar, el ocultamiento natural, logrado sobre la base de las características topográficas y de vegetación, elementos de protección física y visual de la aldea.

Hubo casos en la sierra de Los Órganos, donde el apalencamiento tropezó con la no existencia de áreas para construir un palenque con todas sus especificidades, los que combinaron las habitaciones en cueva y en ranchos al aire libre. En los ubicados al interior de la serranía, cultivaron algunas especies de alimentos, dada la fertilidad de esos terrenos.

Ejemplos de esos tipos de palenques lo constituyen el de Los Ruiseñores, sierra de San Carlos, municipio de Minas; y el palenque de la Sierra de Pan de Azúcar, municipio de Viñales, este último atacado por una partida de rancheadores en noviembre de $1839 .{ }^{2}$

En los manglares del litoral costero, las condiciones de vida y protección no eran las mismas si se le compara con las serranías. Entre las mayores dificultades a las cuales se enfrentaban los cimarrones se hallaban: la carencia de agua potable y las densas plagas de insectos que hacía difícil la vida en tales parajes; pero a la vez, los convertía en lugares donde era posible el aislamiento y resguardo para evitar ser capturado.

La tendencia de la forma de cimarronaje practicada en la franja de manglares fue la cuadrilla. Por lo regular los manglares limitaban tierra adentro con plantaciones cañeras, cafetales y sitios de crianza, convertidas en fuentes de aprovisionamiento sobre la base del robo de alimentos, sobre todo el sacrificio de ganado porcino y vacuno.

El tramo de costa correspondiente al extremo sureste de Vueltabajo, es el de mayor frecuencia en reportes documentales relacionados con la presencia de refugios de cimarrones. Este comportamiento, se debe a lo alejado de estas costas de la Cordillera de Guaniguanico, razón por la cual el cimarrón se veía obligado a penetrar en los manglares, antes de poder alcanzar la serranía.

2 Archivo Provincial de Historia de Pinar del Río. Fondo Instituciones Judiciales Coloniales, $227 / 1380$ 
La llanura cársica de Guanahacabibes presenta como singularidad una topografía cársica con existencia de cuevas y dolinas, algunas de ellas inundadas. Es esta la región, quizás de las cuatro enunciadas, la menos frecuentada por los cimarrones, cuestión lógica, sobre todo para el cimarronaje simple y en cuadrilla, por la resistencia ofrecida por dicha zona al desarrollo económico de la época, y por consecuencia, la limitación de las fuentes de aprovisionamiento.

\subsection{Inevitabilidad del cimarronaje en Vueltabajo}

El cimarronaje durante gran parte del siglo XIX, como fenómeno social del occidente cubano, engrana con el proceso económico e histórico. El auge de la trata, la agudización dela explotacióny elmaltrato impuesto porelnuevomodeloplantacionista, está relacionado con el apogeo y desenvolvimiento de la sociedad esclavista.

La penetración del cultivo de la caña de azúcar y la aparición de un número importante de ingenios en las llanuras, y el florecimiento inusitado de los cafetales, en las zonas montañosas, como ya se indicó, son los efectos palpables del proceso de sustitución de la antigua estructura ganadera, por una nueva conformación agraria, base causal directa del auge de la esclavitud, y por tanto del cimarronaje en esta región de la isla.

Este proceso, en virtud de la necesidad exponencial de mano de obra por los propios requerimientos de la economía, propició un cambio trascendente en el contexto tanto geográfico como social de la región vueltabajera, en la cual se perdió con rapidez, cualquier leve tinte de patriarcalidad de la esclavitud, presente en épocas anteriores, sobre todo en la porción oriental.

A partir de ese momento, la introducción de mano de obra esclava se hizo mayor, más multiétnica, con la agudización de los niveles de explotación, abuso y violencia. Las manifestaciones de resistencia eran por lo tanto inevitables, y también la represión sin cuartel.

Estas expresiones de cimarronaje, se fundamentaron en la evasión de los esclavos hacia sitios aislados al resguardo de la persecución, ya sea de forma individual o en pequeños grupos o cuadrillas; pero no faltaron en esta región las fugas masivas y revueltas e insubordinaciones, ni la existencia de palenques, en zonas críticas, intento de erigirse como territorios libres.

En Vueltabajo, el cimarronaje presenta momentos críticos durante el primer cuarto del siglo XIX, caracterizado por cinco períodos. El primero se enmarca entre 1800 y 1804, con la existencia de los "indios feroces de la Vueltabajo" y su apalencamiento, asociado a un grupo de esclavos africanos, hecho que conmociona y desestabiliza toda la región por varios años. ${ }^{3}$

3 Ver: Armando Abréu Morales. Indios Feroces de la Vueltabajo. Ed. Loynaz, Pinar del Río, 2012 
Entre 1810 y 1811 , se produce el segundo, después del ataque a la familia de Valentín Páez en la comarca de Los Palacios, y su venganza al convertirse en rancheador, momento que culminó con la destrucción del llamado "Gran Palenque de la Vueltabajo".

El tercero fue entre 1814 y 1815 , etapa donde actuaron rancheadores como Matías Pérez Sánchez ${ }^{4}$ y Valentín Páez. Estos dos cazadores de esclavos, efectuaron un intenso rastreo en esta región.

El cuarto es a partir de 1816, y por un tiempo no definido con exactitud, al no aparecer la documentación específica de esa etapa; sólo se habla de ella en referencias posteriores, lo cual induce a pensar en su duración hasta 1817.

La quinta etapa de crisis, la más intensa de las cinco, es la de 1818 a 1825. En esta se inserta, junto a la de otros muchos, la labor de persecución de cimarrones del alférez de Dragones de América, Gaspar Antonio Rodríguez González.

Ello no significa la inexistencia, en épocas o años intermedios, e incluso posterior, de actividades de este tipo. En la década de los años treinta, y principios de la siguiente, se produjeron sistemáticas operaciones de persecución protagonizadas por los rancheadores José Pérez Sánchez y Francisco Estévez.

A partir de una serie de discusiones intensas en la Junta de Fomento en 1819, comenzó un proceso de teorizaciones sobre estrategias y tácticas acerca de cómo combatir la cimarronería. Este grupo de "estrategas" son, desde algunos componentes de la Junta, propietarios, hacendados, como José Rubio Campos, dueño del cafetal La Mariana, ${ }^{5}$ uno de los más participativos en las informaciones y las ideas dadas al gobierno contra los cimarrones; así como el coronel Francisco Chappotín Santo Domingo y el hacendado Antonio Duarte y Zenea.

Este último propietario de ingenios y cafetales, consiliario de la Junta de Gobierno, fue una figura destacada en los aportes para conformar nuevos métodos y estrategias de persecución de cimarrones, tomadas de su experiencia. Los escritos de Duarte y Zenea, junto a los del alférez Rodríguez González, constituyen la base de los cambios realizados en ese sentido por el Gobierno a principios de la década del 20 del siglo XIX.

Para ese momento, las medidas en vigor habían sido bastante ineficaces, al producir muy pocos resultados concretos, y los palenques continuaren en ascenso, hasta el punto de alcanzar mayores niveles a finales de 1819 y principios de 1820 .

4 A principios del siglo XIX era capitán del partido de Cacarajícara. Proporcionó el terreno para la fundación del pueblo de Bahía Honda en 1806. Es uno de los más importantes perseguidores de esclavos en las dos primeras décadas del siglo XIX en Vueltabajo, hermano mayor de quien más adelante se convertiría en el célebre rancheador José Pérez Sánchez.

5 Este cafetal estaba situado en la cuenca del río San Francisco del norte, al pie de las elevaciones de Las Peladas, sierra del Rosario. 
La "impunidad" de los cimarrones, sumado a otra serie de factores, así como la existencia de jefes bien experimentados con un notable poder de decisión, llevaron a concebir la estructuración de grandes grupos circunstanciales, con objetivos bien trazados de ataques combinados sobre haciendas, ingenios y cafetales.

En ese momento ocurrió algo inusual: el paso de la posición defensiva, a una ofensiva en la estrategia de los cimarrones. Hecho significativo, que propicia la agudización de la crisis. En respuesta, se intensificó la actividad del gobierno desde principios de 1820, la cual fue subiendo de tono, hasta alcanzar niveles significativos.

Ante esta situación Rodríguez González, se convirtió en azote para el cimarronaje, y en elemento central de apoyo del gobierno y la oligarquía para resolver este problema crucial.

El gobierno de la Isla había cambiado de manos, José de Cienfuegos dio paso a Juan Manuel Cajigal, hecho coincidente con la intensificación del cimarronaje en Vueltabajo. Las propias ocupaciones del gobierno, sus atenciones a otros aspectos de orden militar y político urgentes y la inestabilidad del momento, entre otros; lo hicieron bajar la guardia. A partir de ese instante, se hizo sentir más la actividad de los cimarrones; quienes comenzaron no a huir y esconderse, sino atacar cafetales y haciendas de manera abierta.

Como respuesta al incremento del cimarronaje, se intensificaron las persecuciones y ataques a rancherías y palenques. El 23 de febrero de 1820, ocurrió un hecho insólito, poco conocido en la historia de la resistencia esclava, cuando se produjo el ataque al palenque de la loma de Los Resbaladeros, en la cuenca superior del río San Cristóbal, efectuado por la combinación de varias partidas, cuyo resultado fue la victoria total y frontal obtenida por los cimarrones.

Las partidas de rancheadores que ejecutaron el ataque a dicho reducto, estaban dirigidas por el capitán pedáneo de Cayajabos, José Garcilaso de la Vega, el mismo que envió al gobierno un informe en enero de 1819, desatador de toda esta persecución, y por el teniente pedáneo de Artemisa, Francisco Fernández, más la partida reunida en Candelaria, bajo el mando de Ramón Cordero Sánchez, quien muere de un certero disparo a consecuencia de la defensa ofrecida por los cimarrones el 23 de febrero de $1820 .^{6}$

Esta situación obligó a las clases dominantes, al gobierno con su poder político y sus graves problemas a cuestas, y a la oligarquía criolla y española, estamentos a quienes perjudicaba la existencia de la rebeldía esclava, a cambiar los métodos y las estrategias para tratar de acabar con el cimarronaje, aunque para ello fuese necesario pasar por encima de los reglamentos ya establecidos. Tal contrariedad era necesaria resolverla, y si los instrumentos dispuestos no eran ya efectivos, había que crear otros nuevos, o ajustar los ya existentes.

6 Archivo Parroquial de San Cristóbal (APSC): Defunciones de Blancos, Libro No.3. 
Revista de Humanidades, 37 (2019).p. 73-98. ISSN 1130-5029

Desde 1816, se debatía con intensidad las vías para combatir la cimarronería, aunque sin llegar a establecer un acuerdo debido al choque de intereses entre los grupos de poder; lo que hizo impostergable la búsqueda de una solución en 1820 . A partir de ese momento, se agudizó la crisis provocada por el cimarronaje, lo que evidencia la expresión de una incongruencia entre el problema y el modo de resolverlo, lo cual ya venía desde atrás y perduraría por un largo período. La solución del fenómeno, se volvió irreconciliable en ese momento histórico, con la estrategia y el método represivo tradicional, que ya no resolvía la situación.

\subsection{Manifestaciones de subsistenciales y defensivas del cimarronaje}

Entre las manifestaciones de subsistencia de los cimarrones en la Región de Vueltabajo ocupaba el lugar más importante, la obtención de alimentos para sus miembros. Sus variantes estaban basadas en tres vías principales: 1) saqueo de haciendas, 2) cultivos de viandas y hortalizas y 3) trueque o comercio en especies.

En el primero de los casos, el saqueo, fue el de más alta incidencia. En los documentos referentes al tema, sobre todo en los diarios de rancheadores, se insiste en la gran cantidad de sustracciones de alimentos en las haciendas. Esta vía era empleada por las cuadrillas y el cimarrón simple; también los apalencados, pero con menor dependencia, efectuaban saqueos.

La obtención de alimentos a través del cultivo de viandas, hortalizas y otras plantas con frutos comestibles, se efectuó en aquellos lugares donde los cimarrones se reunían hasta llegar a establecer palenques. Esto, al no resultar suficiente para mantener al colectivo, los obligaba a buscar otras vías para el complemento alimentario.

El trueque o comercio en especie, se dio entre el cimarrón y algunos administradores y mayorales de haciendas. Esto constituía una vía importante para la obtención de alimentos y resolver otras necesidades. En el intercambio de mercancías, los cimarrones ofertaban miel de abejas, producto que constituía un importante renglón económico en el siglo XIX. A mediados de esta centuria, la región vueltabajera produjo más de dos mil barriles de este renglón para el mercado interno.

Otra vía, aunque no se ha reflejado entre las principales, fue la caza y captura de animales, prácticas indicadoras de una economía natural. La caza de jutías parece haber sido el principal renglón alimentario obtenido.

Sin constituir tendencias, existieron otras formas de relaciones entre el cimarrón y algunos individuos interesados en su uso como fuerza de trabajo. Para el "contratista", este individuo era mucho más barato que el esclavo manso por concepto de manutención. A cambio de ello, el cimarrón recibía el pago en especie y cierta protección. Entre las actividades de este tipo, se puede mencionar el corte y extracción de madera y fibras vegetales. 
La obtención de información sobre los movimientos de los rancheadores y el estado de vigilancia en que se hallaban las haciendas, era un objetivo fundamental de los cimarrones. Resulta interesante el sistema creado por los fugitivos para el logro de tal información, a pesar de su ubicación en lugares recónditos.

Para tener el control del movimiento de los rancheadores, los cimarrones tenían en las dotaciones de esclavos mansos, una segura fuente de datos. Estos se encadenaban con otros esclavos huidos quienes tenían la misión de situarse en lugares topográficamente ventajosos.

En Vueltabajo, se dio con mucha frecuencia otro fenómeno, consistente en la actitud pasiva asumida por algunos hacendados, administradores y mayorales ante la persecución del cimarrón. Esto trajo serias discrepancias entre los rancheadores y dichos individuos.

Una de las astucias de los cimarrones cuando eran perseguidos cerca de determinadas haciendas, era mezclarse con la dotación de algún ingenio o cafetal sin ser notado por su dueño. En el segundo caso, se dio con algunas excepciones, este fenómeno al resultar casi imposible, pues la dotación promedio era de veinte ó treinta esclavos, número fácil de controlar con la consiguiente detección de alguna anomalía como la introducción de esclavos furtivos.

Entre otras tácticas frecuentes estaban los desplazamientos de una región a otra en busca de alimentos y nuevos integrantes, sobre todo mujeres, para los palenques. En la documentación consultada, se nota con reiteración estos flujos migratorios temporales, donde la mayoría de ellos se corresponden con las sierras del Rosario y los Órganos.

El principal foco emisor de cimarrones lo constituyó la subregión plantacionista del extremo oriental de Vueltabajo, en la que se halla situada gran parte de la sierra del Rosario. Es desde estas montañas donde se producía el mayor flujo hacia la sierra de los Órganos y otras subregiones del Occidente, o sea, el movimiento migratorio iba de una subregión, cuyo desarrollo económico era la plantación, hacia una con predominio de economía tabacalera-ganadera.

Esa última subregión con condiciones topográficas no explotables en lo económico, posibilitaba la práctica de la cimarronería, y muy en especial del apalencamiento; aunque esto no quiere expresar que las zonas tabacaleras-ganaderas de Vueltabajo no aportaron al cimarronaje. No pocos esclavos de vegas de tabaco y estancias de labor, asumieron la decisión de convertirse en cimarrones.

También, estas migraciones internas se daban de manera inversa, solo que, con intereses muy específicos, entre ellos, y como ya se hizo referencia, al traslado voluntario o forzado de mujeres para la conformación del palenque y rancherías. De esta manera, los hombres de estas formas organizativas, viajaban a la sierra del Rosario u otros sitios, entre otros propósitos, a buscar mujeres. 
Otras migraciones, aunque en determinadas épocas del año, tenían otras motivaciones, como las relacionadas con el período navideño. Los cimarrones de palenques y rancherías ubicados en otras subregiones, se mezclaban con esclavos mansos de los cafetales de la sierra del Rosario, sobre todo los asentados en el valle del arroyo Manantiales, actual municipio de Candelaria. Ello trajo como consecuencia, la aparición de puntos de reunión o contacto entre ambas elevaciones.

La subsistencia también incluía las prácticas defensivas y de evasión ante el ataque de los rancheadores cuando eran descubiertos los sitios de reunión, ya fuesen palenques, rancherías u otros tipos de asentamientos. Para ello, pusieron en práctica acciones que no por su simpleza dejaron de ser efectivas. Como tendencia emplearon la táctica de, al ser atacados, un reducido número de individuos hacían frente a los agresores, mientras el resto huía a través de vías de auxilio, previstas para tales ocasiones.

En muchos de los casos, los defensores morían en desigual combate o se inmolaban. Cuando eran atacados, tanto cuadrilleros como apalencados, echaban mano a cualquier objeto a guisa de arma. Resulta curioso ver como entre los medios empleados, se hallan los propios elementos provenientes de la naturaleza y existentes en los sitios utilizados como refugios.

El esclavo prófugo fue capaz de incorporar a su acosada vida, conocimientos empírico-espontáneos; del mismo modo fue adaptándose a las condiciones naturales donde se habían refugiado. Los propios elementos de la naturaleza los empleaba a su favor, para evadir la persecución. También la noche constituyó una excelente aliada que, sumado a la agilidad física del fugitivo, le permitía escapar.

\section{CIMARRONAJE FEMENINO EN VUELTABAJO}

La historia de la cimarronería en la región vueltabajera, sobre todo en la primera mitad del siglo XIX, está llena de testimonios documentales de la presencia de la mujer en la práctica del cimarronaje. En el presente artículo, solo se hace mención a aquellos momentos y acontecimientos que marcaron su protagonismo.

El 15 de marzo de 1820, se produjo el ataque combinado al palenque de loma Miracielos, en la cuenca del río Los Palacios. Las partidas estaban dirigidas por el comandante Jacinto Antonio Disdier, militar comisionado para la persecución de cimarrones. Los atacantes chocaron primero con una línea de avanzada, hecho que evidencia, la existencia de una organización o plan defensivo de los apalencados.

Al respecto refiere Disdier:

[...] atacamos á una porción de vigías que tenían apostadas, las que huyendo y dando aviso á sus guerreros, los que se pusieron en defensa sosteniendo un fuego vivo de 
Mujer y cimarronaje en la región histórica... - J. F. Ramirez Pérez y otros

fusil, y una multitud de piedras que continuamente soltaban rodando por la [loma de Miracielos $][\ldots]^{7}$

Véase como resalta aquí la existencia y ubicación en sitios estratégicos de vigías o centinelas, quienes en este caso cumplieron con eficacia su misión. Estos vigilantes eran todas mujeres, indicativo de la existencia de división de funciones en las comunidades cimarronas de la región de Vueltabajo, conducta verificada en ataques a otros palenques.

El 20 de agosto de 1820, el alférez de Dragones Gaspar Antonio Rodríguez González, comisionado para la persecución de cimarrones en Vueltabajo, reportaba que por el testimonio de la cimarrona Agustina, capturada por él y sus hombres días atrás, se sabía era esclava de Francisco Javier Pedroso, dueño del ingenio "San Roque".

Esta cimarrona, partió hacia las elevaciones de la sierra del Rosario en compañía de otros, y se reunió a una cuadrilla. Allí se le solicitó por parte de los jefes cimarrones, Patricio Madam y Fernando Campos, su incorporación al ataque planeado contra el cafetal San Ramón de Agua Claras, propiedad de Francisco Campos, situado en el valle del arroyo Manantiales, ${ }^{9}$ municipio de Candelaria.

La partida capitaneada por el rancheador José Pérez Sánchez, a finales del mes de enero de 1828, realizó una batida por los alrededor del ingenio Santiago, situado al noreste del poblado de San Diego de Núñez, de cuyo resultado capturó a una cimarrona, quien era esclava de la dotación del ingenio La Luisa, y llevaba más de tres meses en estado de cimarronaje. ${ }^{10}$

A comienzos del mes de enero de 1829, la partida de Pérez Sánchez, en combinación con la del capitán del partido de Santa Cruz de los Pinos, Pedro Torres, realizó un ataque al palenque sierra de Escopeta. Antes de efectuar la acción, avistaron una cimarrona situada sobre un promontorio, la cual servía de vigía.

La primera reacción de la mujer centinela, al encontrarse con los rancheadores, fue dar la alarma al resto del palenque. De inmediato, acudieron los hombres para enfrentar a los perseguidores; como resultado de este encuentro los cimarrones se dispersaron, algunos de ellos prefirieron inmolarse y lanzarse por un precipicio. Un rato después, fue hallado el cadáver de una cimarrona al pie del despeñadero. ${ }^{11}$

7 Archivo Nacional de Cuba (ANC):Real Consulado y Junta de Fomento. Leg: 141, No 6934.

8 El mismo hombre que había donado en 1815, tierras del corral Bayate, para fundar el pueblo de Nuestra Señora de la Candelaria, pueblo y municipio de la actual provincia de Artemisa.

9 Arroyo tributario del río Bayate. En el valle de aquel se halla asetado la Villa Turística Soroa y se desprende la Cascada o Salto de Manantiales.

10 Gabino La Rosa y Mirtha T. González: Cazadores de Esclavos. Diarios. Ed. Fundación Fernando Ortíz, La Habana, 2004.

11 Idem., pp.150-151 
En 1835, es capturado el célebre capitán de cimarrones Francisco (Pancho) Mina. A través de sus declaraciones, se puede apreciar el nivel de participación de las mujeres que le acompañaban. Declaró vivir con cuatro varones y tres mujeres cimarronas, todos ellos vivían de manera precaria donde para poder alimentarse practicaban, en lo fundamental, la caza de jutías y majaes.

Las mujeres cimarronas acompañantes eran Gertrudis Conga, esclava de Antonio Duarte y Zenea, María Filomena Carabalí, esclava de la Condesa de Lombillo y Tomasa Criolla. Esta joven de 16 años, es uno de los casos de nacidos en el monte; su madre pertenecía a la dotación del ingenio San Francisco Alfaro. Es un hecho interesante cómo pudo darse este nacimiento, y luego su crecimiento hasta alcanzar esa edad, sin ser capturada por las intensas persecuciones de los rancheadores.

El motivo del desplazamiento de estas mujeres hasta el sitio donde fueron capturadas junto a los otros hombres, estaba vinculado a la fuga del esclavo Pablo Criollo, del cafetal Santa Teresa de Juan Landot, quien les propuso venir a traer cera para trocarla por otros objetos, intercambio muy frecuente entre los cimarrones y residentes blancos.

La incorporación del cimarrón Pablo, al grupo de Pancho Mina, se debió al hecho de llevarle la esclava Rosalía Lucumí, propiedad de Miguel Herrera, devenida en cimarrón, quien la esperaba en el monte para convertirla en su mujer. ${ }^{12}$

En 1837, se organizó otra partida de rancheadores capitaneada por el celebérrimo Francisco Estévez. Por el diario e informes emitidos por este perseguidor de esclavos fugitivos, se puede apreciar la continuidad de la presencia de la mujer cimarrona en la región de Vueltabajo.

E128 de febrero de 1837, Estévezy su partida encontraron un rastro de cimarrones en el río Taco Taco, sierra del Rosario, actual municipio de San Cristóbal. Tras esta pista, se percataron de la imposibilidad de dar alcance a los esclavos evadidos por lo accidentado del terreno; ante esta circunstancia decidieron soltar sus inseparables perros, entrenados en la persecución de cimarrones.

Como resultado de esta "cacería", llegaron al pie de un farallón donde se apreciaba una cueva en lo alto, a la cual subieron no sin muchas dificultades. Para acceder a ella fue necesario encender una luz en cuyo interior, capturaron una cimarrona. Al ser interrogada expresó ser esclava de la dotación del ingenio La Luisa, municipio de Bahía Honda, y que marchaba acompañada de otra mujer y un varón del mismo ingenio. ${ }^{13}$

La causa de su incursión por aquella zona, se debía que sus compañeros, tenían la intención de conducirla a un palenque situado más al oeste, otra muestra

12 ANC: Gobierno Superior Civil, 616/19688

13 Por esta cimarrona, Estevéz cobró en marzo de 1837, 4 reales. 
de la captación y conducción voluntaria de mujeres hacia palenques y rancherías. ${ }^{14}$ Este fenómeno se repetía de manera sistemática. Años después, Estévez volvió a mencionar a una esclava del cafetal San Andrés, ubicado en la sierra del Rosario, y su sospecha de haber sido raptada por una cuadrilla, motivo por el que "[...] suelen bajar de tiempo en tiempo a hacer sus presas [las mujeres]"15

El 19 de marzo de 1837, la partida de rancheadores de Estévez, al seguir una huella de cimarrones, los condujo a la cuenca del río San Francisco, municipio de San Cristóbal, donde hallaron un palenque, donde fue imposible capturar a ninguno de los miembros, por ser una zona muy accidentada.

En esa operación encontraron una niña recién nacida, hija de una de las cimarronas del palenque, quien, en medio de su huida, no pudo llevarla consigo. Estévez, se quedó a cargo de la criatura al considerar tener derecho sobre ella, debido a que tiempo atrás se le había escapado una esclava embarazada, por lo cual suponía ser gestada por la fugitiva. ${ }^{16}$

El 6 de mayo de 1837, Estévez capturó al jefe de cimarrones Antonio José, quien el día 17 del propio mes y año, le sirvió de guía hasta el sitio donde tenía su palenque, situado en la cima de la sierra de San Juan de Guacamayas, al sureste del poblado de Bahía Honda. Ese día cayó en su poder una cimarrona que resultó ser de su propiedad. ${ }^{17}$

Como consecuencia de una intensa batida ejecutada por la partida de Estévez, en el mes de septiembre de 1837, se entregaron algunos cimarrones, entre ellos el afamado jefe Juan Portugués, quien había estado en las montañas por mucho tiempo. Como parte de esas presentaciones también se entregó la madre Pastora, mujer del jefe Mariano Gangá, muerto en un enfrentamiento con los rancheadores. ${ }^{18}$

La fuga de mujeres hacia palenques y rancherías se intensificó en 1838. Estévez reporta que el 3 de septiembre, fue atacada una cuadrilla de cimarrones en un sitio que ellos denominaban Mavengue de funda de vaca. En esa ocasión, capturaron una cimarrona esclava del Barón de Kessel.

El 17 de septiembre de 1838, la partida de Estévez atacó un palenque situado en la zona conocida como Abra Venturosa, cuenca superior del río Los Palacios. Como resultado de este hecho murieron varios cimarrones y capturaron una mujer, esclava del inmigrante y caficultor vasco Pedro Soroa, quien poseía el cafetal San José, al norte de la loma del Mulo, territorio del actual municipio de Bahía Honda. ${ }^{19}$

\footnotetext{
14 Villaverde, Cirilo: Diario del rancheador. Ed. Letras Cubanas, La Habana, 1982, pp.43-44

15 Idem., p. 110

16 Idem., pp.46-47

17 Idem., p.51

18 Idem., p.67

19 Idem., p.84
} 


\subsection{El caso de la jefa de cimarrones Madre Melchora}

El papel jugado por las mujeres como jefas de palenques o cuadrillas, es evidencia de que en algunos casos tenían una fuerte influencia dentro de las comunidades cimarronas, tanto en Cuba como en el resto de las colonias americanas. Existieron jefas que alcanzaron notoriedad, entre ellas: Romaine "La Profetisa", Marie Jeanne y Henriette Saint-Marc en Haiti; en Jamaica, Nanny Town, de gran ascendencia dentro de las luchas de los esclavos en esa isla; en Estados Unidos de Norteamérica, Sarah y Zeferina en Brasil (Thomson, 2005).

En Cuba, específicamente en la región de Vueltabajo, el caso emblemático es el de la jefa de cimarrones Madre Melchora, quien alcanzó notoriedad en la primera mitad del siglo XIX. Su actividad al frente de un palenque, fue reportada por primera vez en el diario del capitán de rancheadores Francisco Estévez, en el mes de octubre de 1838. En la documentación consultada sobre la presencia de cimarrones en dicha región, no aparece otroejemploen que una esclava hayasido cabeza de una cuadrilla de cimarrones.

Después de la publicación del diario de Estévez, algunos investigadores han considerado a la Madre Melchora como una leyenda -resultado de la imaginación popular-criterionocompartidoporlos autores. Lasmujerescimarronas, fueronenérgicas en su condición de evadidas, aun cuando incluso, desafiaban la costumbre africana que imponía la condición masculina por encima de todo, sometiéndola a sus antojos.

En su primer reporte, Estévez relata haber localizado en las cabezadas del río Santa Cruz, actual municipio de San Cristóbal, provincia cubana de Artemisa, la cuadrilla de la Madre Melchora, compuesta de unos cuarenta cimarrones. En el enfrentamiento sostenido con sus integrantes, solo lograron malherir a tres individuos, el resto logró escapar por haberse descubierto a tiempo la presencia de los rancheadores. ${ }^{20}$

A partir de este primer reporte, en el diario de Estévez aparecen con frecuencia informes acerca de la presencia de la Madre Melchora y su cuadrilla, para ese momento se había convertido en una obsesión la captura de aquella valerosa cimarrona. El 13 de noviembre de 1838, se indica su posible presencia en la cuenca superior del río San Francisco municipio de San Cristóbal. ${ }^{21}$

Una fracción de la partida de Estévez, localizó el 20 de noviembre, la cuadrilla de Melchora en los farallones de la Faranda, elevaciones situadas en la vertiente este de la mencionada cuenca. De este encuentro resultó muerto uno de los cimarrones. ${ }^{22}$

En la continuidad de su incesante persecución, Estévez capturó un cimarrón en el río Taco Taco el 15 de diciembre de 1838, quien le informó estar cerca del palenque de la

\footnotetext{
20 Idem., p. 88

21 Idem., p.89

22 Idem., p.91
} 
Madre Melchora, compuesto por treinta y seis cimarrones. Al siguiente día, decidieron atacar el enclave rebelde, el que encontraron vacío por estar avisados sus integrantes. ${ }^{23}$

Esemismo día, en el sitio conocido como la Fuente de Peña Blanca, son capturados dos cimarrones. En su confesión, declararon que la mayoría de los palenques estaban situados más al occidente, y solo el de la Madre Melchora, operaba en la zona de las cabezadas del río Santa Cruz. Este elemento, le concede mayor relevancia a la valentía de esta jefa de cimarrones, al ser esta región muy controlada por el aparato represivo de los hacendados, dada su proximidad a las haciendas cafetaleras e ingenios. Para una cuadrilla de cimarrones, resultaba en extremo difícil mantenerse en dicha zona, de ahí la continua movilidad a la que tanto se refiere Estévez. ${ }^{24}$

La partida de Estévez, capturó el 6 de febrero de 1839, a dos cimarrones refugiados en una antigua ranchería en la zona de Rangel, cuenca superior del rio Taco Taco, municipio de San Cristóbal, donde llamaban Mavengue del guanajo. Uno de ellos auxiliaba a un cimarrón por indicaciones de la Madre Melchora, herido de bala en encuentros anteriores. El hecho muestra el lado sensible y la responsabilidad de esta jefa por los integrantes de su cuadrilla.

Tras este encuentro, la partida de rancheadores continuó la persecución, hasta que el día 10 de febrero en el Mogote del Mono, destacada elevación situada a un lado del camino de Rangel, al noroeste del actual poblado de Santa Cruz de los Pinos, localizaron la cuadrilla de la Madre Melchora. En esos momentos, sus integrantes estaban levantando una nueva ranchería, hallándose desparramados, en busca de guano y yaguas para cubrir los ranchos, hecho que impidió la sorpresa. No obstante, los rancheadores capturaron a tres de sus miembros. ${ }^{25}$

Todo parece indicar que el persistente acoso de la partida de Estévez, tras la cuadrilla capitaneada por la Madre Melchora surtió efecto, pues después del encuentro en el Mogote del Mono, no se supo más de la legendaria cimarrona, quien no pudo ser capturada, a juzgar por la documentación hasta ahora consultada.

En octubre de 1839, un cimarrón capturado declaraba que debido al constante acoso, las cuadrillas de cimarrones habían decidido refugiarse más al occidente, ${ }^{26}$ hacia la sierra de los Órganos, lo cual hace posible el desplazamiento de la Madre Melchora hacia esa zona.

Resulta sintomático que en noviembre de 1839, un mes después del traslado de las cuadrilla de cimarrones más al occidente, una partida de rancheadores comandada por Estanislao Ribera ${ }^{27}$ atacó un palenque en la sierra mogótica de Pan de Azúcar,

23 Idem., p.93

24 Idem.

25 Idem., pp.94-95

26 Idem., p.105

27 Este capitán de rancheadores, era vecino del partido de Consolación del Norte. Su partida estaba integrada, entre otros hombres, por algunos miembros de su familia. Alcanzó notoriedad en la 
actual municipio de Viñales, y capturó una cimarrona la cual mencionó la existencia de otra mujer en el palenque, llamada Melchora Carabalí, quien no pudo ser capturada. ${ }^{28}$

Este dato es un muy interesante, el nombre de Melchora no se repite mucho entre las mujeres esclavas durante el periodo de auge del cimarronaje en Vueltabajo. Al tenerse en cuenta la fecha de reporte del palenque de Pan de Azúcar, y el nombre de la mujer escapada de la persecución del rancheador Ribera, pudiera ser la misma Madre Melchora, quien continuó su bregar en las montañas vueltabajeras. Hasta el presente, su destino final es una incógnita.

\subsection{El caso de la cimarrona Petrona Conga}

Como ya se ha dicho, fue práctica de los cimarrones varones, el rapto o la huida forzada de mujeres para llevarlas a los palenques y rancherías por diversas razones, ya sea para convertirlas en compañía de alguno de sus integrantes masculinos, o para incrementar su número al reconocer, el valor demostrado por estas en el enfrentamiento a sus adversarios. Un hecho de esta naturaleza, ocurrió en el mes de noviembre de 1839, al ser capturada la cimarrona Petrona Conga.

Los hechos ocurrieron cuando el capitán de rancheadores, Estanislao Ribera, detectó huellas de cimarrones. En compañía de sus hombres se precipitó tras ellos, el 11 de noviembre de 1839, hasta localizar la ranchería en el centro de la sierra de Viñales, a la cual ascendieron desde la zona de Pan de Azúcar. El primer contacto con los rebeldes ocurrió a la entrada del palenque, donde estaban apostadas las mujeres que servían de vigías.

Al percatarse de la presencia de los rancheadores, tomaron la decisión de evacuar la posición, a lo que respondieron los perseguidores con algunos disparos de advertencia. De inmediato, emprendieron una carrera en su persecución, al llegar a un despeñadero lograron alcanzar a una de las cimarronas, nombrada Petrona Conga, mientras el resto logró escapar.

Con Petrona prisionera, los rancheadores volvieron a la ranchería, para interrogarla. En su información dijo que eran en total dieciocho personas, entre ellas quince hombres y tres mujeres, que, en el momento del asalto, la mayoría de los hombres habían bajado de la sierra a buscar carne de cerdo y res, por lo que en la ranchería solo habían quedado tres mujeres y dos hombres.

En su testimonio abundó diciendo que los de la ranchería tenían, además, el propósito de ir al cafetal Siberia, de Pascual Pluma, en la zona de San José de Manantiales (Soroa), actual municipio Candelaria, a buscar más negros y provisiones.

Jurisdicción de Nueva Filipina, por sus correrías detrás de los cimarrones.

28 Archivo Provincial de Historia Pinar del Río (APHPR). Fondo: Instituciones Coloniales. 
Otro objetivo del grupo de cimarrones, una vez consolidados, era ubicar las casas de interés, o sea, aquellas con tabacos, cera y prendas para saquearlas.

El 16 de diciembre de 1839, en la cárcel de la ciudad de Pinar del Río, Petrona fue sometida a un nuevo interrogatorio. Era natural de África, casada y esclava de Carlos Kessel, de la dotación de un cafetal (Siberia) de éste, situado en la zona de San José de Manantiales.

La historia de esta esclava resulta muy interesante, para comprender como estas mujeres se convertían o eran convertidas en cimarronas. En 1838, al oscurecer de un día, después de haber concluido la jornada de trabajo, regresó a su bohío, y de inmediato, salió de la habitación para dar de comer a unos cerdos. A la mitad del camino, se encontró con cinco supuestos esclavos africanos, a los cuales confundió con compañeros suyos. Al acercarse, se percató de que no pertenecían a la dotación, por lo cual intentó retirarse.

En ese momento, uno de los cimarrones, le explicó que habían venido a buscarla para conducirla a donde se encontraba su marido. Ante esta situación reflexionó y llegó a pensar que su esposo podía estar entre los apalencados, pues el mismo hacía algunos días que su amo lo había transferido a un ingenio y era probable, el haberse evadido por su inconformidad con la medida.

El deseo de unirse a su cónyuge la llevó a creerles a aquellos hombres, y fue hasta el bohío a buscar ropa. Ya de regreso comenzó a dudar, y temerosa de ser engañada decidió retroceder, pero el jefe de aquel grupo de cimarrones, la agarró por una mano y la amenazó con que si no le seguía la mataría. Ante el temor y sin otra alternativa, se entregó a los deseos de sus captores. En su compañía, emprendió el camino, primero a lo largo de la sierra del Rosario y luego por la sierra de los Órganos, hasta llegar a la zona de Pan de Azúcar, en un recorrido de más cien kilómetros.

Según Petrona, en la ranchería había dos mujeres más: Inés Conga, esclava del varón de Kessel y Melchora Carabalí, de la cual ignoraba quien era su dueño. Esta última cimarrona es el caso comentado en el acápite anterior ¿Será la misma capitana de cimarrones quien operó en la sierra del Rosario?

El lograr sobrevivir estas mujeres, junto a los hombres del palenque, era a partir de la sustracción de alimentos de las haciendas, en su gran mayoría, cerdos, propósitos logrados al incursionar, de modo alterno, en partidas de tres o cuatro hombres, pues a las mujeres no les permitían salir de los ranchos. Su trabajo en el palenque, además de sus obligaciones como pareja, era la de vigilancia, cuestión extendida en estas condiciones de acoso. Como se aprecia, esto es un testimonio en alto elocuente, de la vida de una cimarrona. ${ }^{29}$

29 APHPR. Fondo: Instituciones coloniales 
Revista de Humanidades, 37 (2019). p. 73-98. ISSN 1130-5029

\section{CONGLUSIONES}

Estos dos casos, más los reportes de mujeres cimarronas mencionados, son suficientes argumentos para atestiguar la presencia femenina en el fenómeno del cimarronaje en la región histórica de Vueltabajo, por un largo periodo de tiempo. Cada una de ella, es testimonio de cuanto debieron sufrir en su condición de esclavas en las dotaciones de las diferentes haciendas existentes en ese escenario geográfico, para verse en la obligación de evadirse de ellas.

Pesquisas más profundas sobre la participación femenina en la cimarronería, no solo en la región vueltabajera, sino también en todo el archipiélago cubano, pudiera develar la magnitud de esta participación. El escaso reconocimiento a la mujer cimarrona en la historiografía cubana, alienta otras miradas hacia la perspectiva de género en la rebeldía esclava, como portadoras y trasmisoras de una cultura de resistencia.

\section{BIBLIOGRAFIA}

Abreu Morales, Armando (2012). Indios Feroces de la Vueltabajo. Pinar del Río: Loynaz.

Abreu Morales, Armando. Historia de La Palma. Periodo Colonial. Museo Municipal. La Palma. [Inédito].

Alonso Alonso, Enrique (1986). Encuentro con el Cimarrón del Río Santa Cruz. Trabajo presentado en el evento por el Centenario de la Abolición de la Esclavitud, Matanzas. [Inédito].

Estado Mayor General del Ejército Español (1854). Sección Mariscales de Campo. Madrid: Imprenta de T. Fortanet.

Formigo Espinosa, Luís y Márquez Jaca, Sergio Luís. El alférez de Dragones Gaspar Antonio Rodríguez: rancheador, conspirador y pirata. [Inédito].

Franco, José Luciano (1967). Cuatro Siglos de lucha por la Libertad: Los Palenques. En: Revista de la Biblioteca Nacional José Martí, 58 (1), pp. 5-44.

Franco, José Luciano (1982). Los palenques de los negros cimarrones. La Habana: DOR del CC-PCC.

González, María Teresa (1988). Aproximaciones y diferencias entre los hacendados y el Código Negro Español de 1789. En: Temas acerca de la esclavitud. La Habana: Ciencias Sociales.

Guerra, Ramiro (1973). Manual de Historia de Cuba. La Habana: Ciencias Sociales.

Márquez, Sergio Luis y Ramírez, Jorge Freddy (1990). El Cimarrón del Río Santa Cruz: estudio histórico-antropológico. En: Albur: revista cultural cubana (órbita).

Núñez Jiménez, Antonio (1976). Isla de Pinos: piratas, colonizadores, rebeldes. La Habana: Arte y Literatura. 
Mujer y cimarronaje en la región histórica... - J. F. Ramirez Pérez y otros

Ramírez, Jorge Freddy (1986). Impotencia de las partidas de rancheadores ante el fenómeno de la cimarronería en Vueltabajo. Trabajo presentado en el evento por el Centenario de la Abolición de la Esclavitud, Matanzas. [Inédito].

Ramírez, Jorge Freddy y Márquez, Sergio Luís (1988). Diario de operaciones de un rancheador militar en Vueltabajo. En: Alcance a la Revista de la Biblioteca Nacional, 2 (2).

Ramírez, Jorge Freddy y Hernández Pérez, Pedro Luís (2005). Regionalización paisajística de la resistencia esclava en Vuelta Abajo. Trabajo presentado en el Congreso Nacional de Historia. [Inédito].

Ramírez, Jorge Freddy (2010). Perros para cazar esclavos africanos. En: Cauce (2), Pinar del Río.

Rosa, Gabino de la (1986). Los Palenques en Cuba: Elementos para su reconstrucción histórica. En: La esclavitud en Cuba. La Habana: Academia de Ciencias de Cuba.

Rosa, Gabino de la (1987) Cimarrones, apalencados y rancheadores en Puerto Príncipe. En: Del Caribe (Santiago de Cuba) 4 (8).

Rosa, Gabino de la (1991). Los Palenques del Oriente de Cuba. Resistencia y acoso. La Habana: Academia.

Rosa, Gabino de la y González, Mirtha T. (2004). Cazadores de esclavos: diarios. La Habana: Fernando Ortiz.

Thomson, Alvin O. (2005). Huida a la libertad. México: Siglo XXI.

Villaverde, Cirilo (1982). Diario de un Rancheador. La Habana: Letras Cubanas.

\section{FUENTES DE ARGHIVO}

\section{ARCHIVO NACIONAL DE CUBA}

Fondo: Real Consulado y Junta de Fomento. Legajo: 141, Nº 6940.

Fondo: Comisión Militar. Leg: 1, №.2

Fondo: Real Consulado y Junta de Fomento. Leg: 141, № 6934.

Fondo: Gobierno Superior Civil. Leg: 625, № 19876.

Fondo: Real Consulado y Junta de Fomento. Leg: 150, № 7416

Fondo: Gobierno Superior Civil. Leg: 625, ํ 19876

Fondo: Real Consulado y Junta de Fomento. Leg 150, №. 7421

ARCHIVO PROVINGIAL DE HISTORIA, PINAR DEL RIO

Fondo: Instituciones Judiciales Coloniales, Leg.227, №.1380

ARCHIVO PARROQUIA DE SAN CRISTOBAL

Fondo: Libro Defunciones de Blancos, No.3. 